\title{
The complexity and difficulty of diagnosing lung cancer: findings from a national primary-care study in Wales
}

\author{
Richard D. Neal ${ }^{1}$, lain J. Robbé ${ }^{2}$, Malcolm Lewis ${ }^{3}$, lan Williamson ${ }^{4}$ and Jane Hanson ${ }^{5}$ \\ ${ }^{1}$ North Wales Centre for Primary Care Research, Bangor University, Wrexham, UK \\ ${ }^{2}$ Clinical Assistant Professor, Division of Community Health and Humanities, Faculty of Medicine, Memorial University \\ of Newfoundland, Canada \\ ${ }^{3}$ Director Postgraduate Education for General Practice, School of Postgraduate Medical and Dental Education, \\ Cardiff University, Cardiff, UK \\ ${ }^{4}$ Consultant Chest Physician, Chair of the Lung sub-group, Cancer National Specialist Advisory Group, Southampton, UK \\ ${ }^{5}$ Lead Adviser for Cancer, Welsh Government, Head of Cancer National Specialist Advisory Group Core Team, Cardiff, UK
}

\begin{abstract}
Aim: This paper aims to provide a detailed analysis of the diagnostic process of lung cancer from a primary-care perspective. Background: Diagnosing lung cancer at a stage where curative treatment is possible remains a challenge. Beginning to understand the complexity and difficulty in the diagnostic journey should enable the development of interventions in order to facilitate timelier diagnosis. Methods: A national study of significant events was conducted whereby general practitioners (GPs) in Wales were asked to report data relating to the diagnostic process of recent lung cancer diagnoses using a standard template. Both qualitative and quantitative data were analysed. Findings: Case reports were received from 96 general practices on 118 patients. A total of 96 patients (81.4\%) presented with respiratory symptoms. A total of 79 patients $(66.9 \%)$ had a GP-initiated X-ray before diagnosis. A total of 23 patients (19.5\%) had a chest X-ray that did not initially show suspicion of lung cancer. A total of 25 patients (21.2\%) were diagnosed after a GP-initiated acute admission. Analysis of free-text qualitative data showed that, for many patients, their GP behaved in an exemplary manner. However, for some patients, the GP could have made more of the opportunities presented for timelier diagnosis. There were a number of atypical and complex presentations, where the opportunities for more timely diagnosis were more limited. A variety of causes of diagnostic delays in secondary care were reported. These findings will inform health policy, and will inform the design of interventions to try to facilitate more timely diagnosis for symptomatic patients. We encourage greater compliance with diagnostic guidelines and greater vigilance for patients presenting with atypical symptoms, as well as for patients whose initial chest X-rays are normal.
\end{abstract}

Key words: diagnosis; lung cancer; primary care; significant event

Received 13 August 2013; revised 21 October 2014; accepted 28 October 2014;

first published online 8 December 2014

\section{Introduction}

Lung cancer is one of the commonest cancers in the developed world and has poor survival rate

Correspondence to: Professor Richard D. Neal, North Wales Centre for Primary Care Research, Bangor University, Wrexham Technology Park, Wrexham LL13 7YP, UK. Email: r.neal@bangor.ac.uk
(Office for National Statistics, 2010). This is because most patients are diagnosed at a stage when curative treatment is not possible (Neal and Muers, 2009). Although the search for diagnostic molecular biomarkers and the assessment of screening programmes continues, neither of these is currently available. In the UK, most patients will initially present, with symptoms, to general 
practitioners (GPs) working in primary care (Barrett and Hamilton, 2008; Elliss-Brookes et al., 2012). There is a need to focus on the diagnostic processes for people who present with symptoms, because there are potentially missed opportunities to diagnose them earlier (O'Dowd et al., 2014). Lung cancer is one of the hardest cancers to diagnose, and there are many pitfalls in making a diagnosis (Neal et al., 2014). More than a third of patients consult three times or more before referral (third highest of 18 cancers) (Lyratzopoulos et al., 2013), and 39\% of lung cancers present as an emergency (only brain and pancreatic cancers are higher) (Elliss-Brookes et al., 2012). Both of these are markers for poor outcomes.

Recent articles have reported time intervals in the diagnostic pathway. The median time from onset of symptoms to presentation was 12 days [interquartile range (IQR) 0-33 days] (Keeble et al., 2014). The median time from first presentation to diagnosis was 112 days (IQR 45-251); this was the second highest of 15 cancers (Neal et al., 2013). The median time from first being seen in primary-care to referral for specialist opinion was 14 days (IQR 3-40), and this increased with increasing numbers of pre-referral consultations; only myeloma was higher (Lyratzopoulos et al., 2013).

GPs currently work within the framework of the National Institute for Heath and Care Excellence (NICE, 2005) guidelines. This mandates referral for a chest X-ray for certain symptoms, and urgent referral for others. These guidelines are in the process of being revised.

In 2008-2009, as a part of the National Awareness and Early Diagnosis Initiative (NAEDI) in England, the North of England Cancer Network (NECN) studied cancer diagnoses in primary care by undertaking a Significant Events Audit (SEA) (Mitchell et al., 2009; 2013). They reported 132 diagnoses of lung cancer, within the context of the English National Health Service (NHS), and have called for more research to better describe the diagnostic pathway for lung cancer following presentation.

We replicated the NECN study in Wales for two reasons. First, because Wales has poor lung cancer outcomes compared with elsewhere. The resection rate for all non-small-cell lung cancer (NSCLC) was $9.8 \%$ for Wales, compared with $13.7 \%$ for England and Wales (NHS Information Centre, 2011). Second, because NHS Wales is organisationally different from England, with Health
Boards having responsibility for community care, primary care, and secondary care, and thus the diagnostic pathways are different. This paper aims to provide a detailed analysis of the diagnostic process of lung cancer from a primary-care perspective, using both quantitative and qualitative data.

\section{Methods}

\section{Data collection}

In January 2010, all 486 general practices in Wales were invited to participate. GPs were asked to complete an electronic template regarding the most recent one or two diagnoses of lung cancer in their practice. The data collection template is shown in Appendix A. It was identical to the one used by NECN (Mitchell et al., 2009; 2013), which in turn was based upon the structure for SEA recommended by the National Patient Safety Agency (National Patient Safety Agency, 2006). A significant event can be applied to any aspect of healthcare and can be applied to either a 'good' or 'bad' event. In relation to this study, the 'event' was a diagnosis of lung cancer. GPs were incentivised to participate as this was part of a Locally Enhanced Service (£100/case report).

In this paper, we present the data that were recorded in the two fields that related to the diagnostic process. They were 'What happened?' and 'Why did it happen?'. Analysis of other data within the templates ('What has been learned?', 'What has been changed', and 'What was effective about this SEA'?) are reported elsewhere (Neal et al., 2010). Our reporting is in keeping with recent consensus guidelines for research into symptomatic cancer diagnosis (Weller et al., 2012).

\section{Definitions}

All of the data, with the exception of factual data relating to the practice, were narrative. Key data fields from the narrative data were extracted either verbatim from the text or with clinical interpretation from the text.

'First presented symptom(s)': these were categorised into either 'respiratory' or 'non-respiratory' in the same way as the NECN report (Mitchell et al., 2009). Some symptoms were not included in their categorisation; therefore, lump in neck, hoarseness, shoulder pain, costal margin pain, and rib pain were 


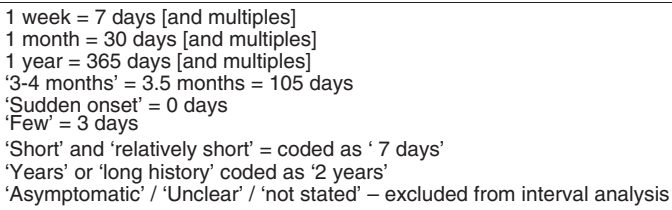

Figure 1 Calculating patient intervals

included as respiratory symptoms. Patients who were asymptomatic were excluded from this particular analysis, as were patients whose symptoms were 'unclear' or 'not stated'.

'Patient interval' (time from symptom onset to presentation): a two-year cut off was taken as the maximum duration of symptoms before presentation. We chose this because it is established that patients with lung cancer often report symptom duration of greater than one year before diagnosis (Corner et al., 2005). Although other studies have examined data from only up to one year before diagnosis (eg, Hamilton, 2009), we opted for the longer period so that no potential symptoms were missed. When one or more different symptoms with different durations were recorded, the duration was recorded as the longer value; the durations were re-coded into days (Figure 1).

'Number of consultations before referral or investigation': this included all primary-care contacts (face-to-face consultations, nurse consultations, telephone consultations, out of hours, home visits) before initial referral or investigation request that could reasonably have been expected to lead to a diagnosis. In three instances, the GP had simply reported 'several'; these were re-coded as ' 3 '. One had recorded 'frequent'; this was re-coded as ' 12 ' (i.e., monthly). Others were either unclear or were not stated; these were excluded from this analysis.

'Date of request of first GP-initiated chest X-ray and date report received': these were recorded verbatim or calculated from the text (eg, report received two days later).

'Referrals and admissions': a referral was regarded as such where there was clear evidence of a GP referral to a specialist. The specialties were mutually exclusive, and coded as either 'chest clinic/respiratory physician', 'ear nose and throat', 'gastroenterology', 'neurology', 'rheumatology', or 'not stated'. Urgency of referral was sometimes mentioned but not in a systematic way; therefore, no further analysis of this was possible. Admissions were coded as either 'medical admission', 'surgical admission', or 'spinal team admission'.

'Date of diagnosis': these were entered as provided by the GP. It was clear that sometimes the date of diagnosis related to a clinical diagnosis, a computerised tomography (CT)/positron emission tomography diagnosis, a tissue diagnosis, or a diagnosis after completion of staging. There were occasional inaccuracies within these dates and a small number was necessarily amended - for example, when the GP stated date of abnormal chest X-ray as the date of diagnosis, but when the patient went on to have further definitive diagnostic procedures. A 'higher level' of date of diagnosis was, therefore, used when possible, in keeping with reporting recommendations (Weller et al., 2012).

'Diagnostic pathways': after reading and re-reading all the reports, each patient was then classified into one of the 11 mutually-exclusive pathways (reported in Table 2).

'Time intervals to diagnosis': from the data provided, eight specific time intervals were calculated (Figure 2). However, because the data were provided in an unstructured narrative format, some data points were missing, meaning that time intervals could not be calculated for the entire sample.

\section{Quantitative data analysis}

All of the quantitative data are presented descriptively, using both parametric and non-parametric statistics as appropriate.

\section{Qualitative analysis of the diagnostic journey}

The data were analysed using a modified Framework approach (Gale et al., 2013). One researcher led the analysis in discussion with the other members of the research team. Therefore, our approach was similar to the analysis of the NECN report (Mitchell et al., 2009; 2013). Although we were aware of the findings from the English study, our analysis was carried out de novo. Quotations are reported verbatim to illustrate the key themes.

\section{Results}

\section{Practices, patients, and tumours}

Reports were received regarding 118 patients from 96 general practices (Table 1). Among them, 


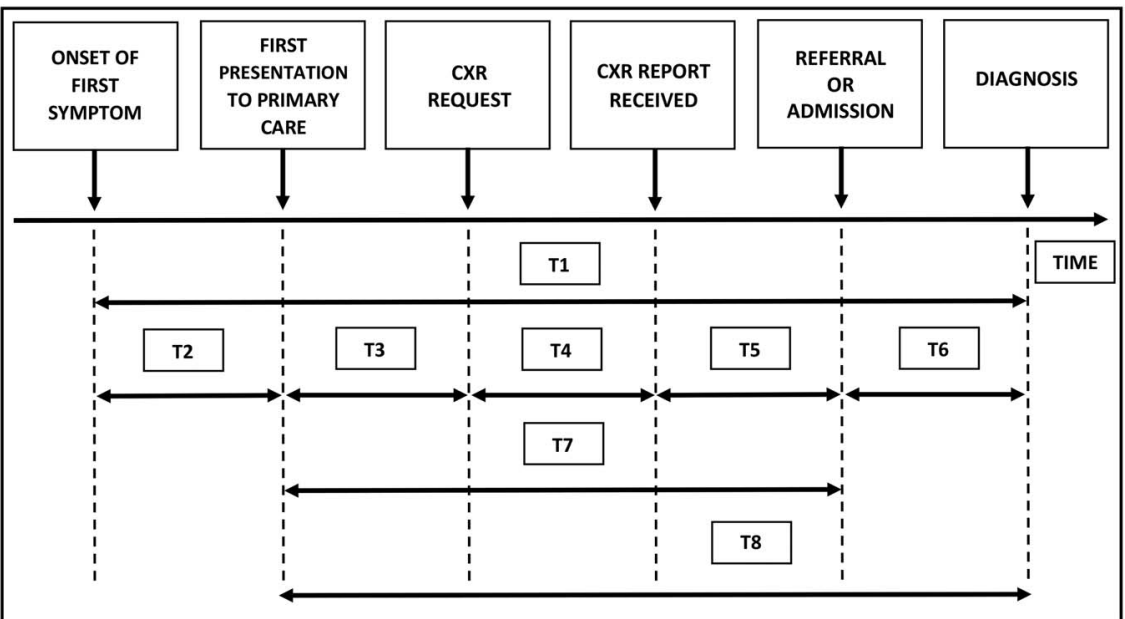

Figure 2 Schematic representation of the time intervals from initial presentation to diagnosis

Table 1 Participating GPs by health board

\begin{tabular}{lllc}
\hline Regional cancer networks & Local health boards & $\begin{array}{l}\text { Number of general } \\
\text { practices per local health board }\end{array}$ & $\begin{array}{c}\text { Number of case } \\
\text { reports received }\end{array}$ \\
\hline North Wales & Betsi Cadwaladr University & 121 & 29 \\
South East Wales & Aneurin Bevan & 94 & 15 \\
& Cardiff \& Vale University & 70 & 24 \\
Couth West Wales & Cwm Taf & 52 & 10 \\
& Abertawe Bro Morgannwg & 77 & 20 \\
Hywel Dda & 55 & 14 \\
& Powys Teaching & 17 & 6 \\
& & 486 & 118 \\
\hline
\end{tabular}

50 practices $(46.9 \%)$ described themselves as 'urban', $29(30.2 \%)$ as 'semi-rural', and $16(16.7 \%)$ as 'rural' (data missing for two practices). Seven $(7.3 \%)$ had a list size of $<2500,14(14.6 \%)$ of $2500-5000$, and $73(76.0 \%$ ) of $>5000$ (data missing for one practice). A total of $45(46.9 \%)$ were training practices and $57(59.4 \%)$ were teaching practices (data missing for one practice). Most of the patients in the 118 case reports were diagnosed recently (24 in 2010, 77 in 2009, 12 in 2008, and 5 in 2007 or earlier). The diagnoses reported covered the following cancer types: 90 were NSCLC without evidence of metastases; 11 were NSCLC but with evidence of metastases at diagnosis; 15 were small-cell cancers; one was a neuro-endocrine tumour; and one was a mesothelioma. Mean age at diagnosis was 69 years. Data were analysed and reported for all 118 patients except where indicated.

\section{Qualitative analysis}

Our findings are reported under three main themes. The first of these is 'GPs' responses to symptoms' - this presents the range of responses from exemplary behaviour to situations where things could have been done differently to difficulty of complex and atypical presentations. The second is 'GPs' perceptions of patients' responsibility for delaying the diagnostic process' - this presents the range of patient-related behaviours that impact upon the diagnostic process. The third is 'GPs' perceptions of secondary-care responsibility for delaying the diagnostic process' - this presents the 
range of secondary-care-related behaviours that impact upon the diagnostic process.

\section{GPs' responses to symptoms}

\section{Exemplary practice}

For a large number of case reports, the response of the GP to the presented symptoms, working from the data provided on the report forms, were exemplary. That is, that there was an appropriate and timely action (chest X-ray, admission, or urgent referral) in response to symptoms, in line with current NICE guidance (NICE, 2005).

'Exemplary GP behaviour. Exemplary radiology behaviour (direct referral for $C T$ after abnormal CXR [Chest X-Ray])'.

(Case \#133: age 71, presenting with cough and pink sputum of 28 days' duration)

A smaller number of patients had 'almost-exemplary' primary-care behaviour. For example, this included patients referred for chest X-ray, investigation, or opinion within a few weeks of symptoms, but outside of current NICE guidance (NICE, 2005).

'On reviewing the NICE guidelines it is evident that this lady should have ideally been referred on presentation since she had cough, headache and hoarse voice for $>3$ weeks, however the referral was only delayed 1 week which we don't think would have significantly affected prognosis'.

(Case \#53: age 62)

Opportunities for more timely diagnosis

For a number of patients, it was clear that there were opportunities to consider a chest X-ray earlier.

'3 previous consultations were for chest infections/cough. Diagnosis made via ENT. GPs wonder whether they should have organised CXR at time of ENT referral'.

(Case \#64: age 49, with sore throat and respiratory symptoms of 90 days' duration)

'Initial presentation with haemoptysis - explained "viral" - 3 week delay until presented again'.

(Case \#76: age 67)

'In retrospect GPs unsure whether could/ should have sent for CXR earlier'.

Primary Health Care Research \& Development 2015; 16: 436-449
[Case \#47: age 75, with frequent exacerbations of COPD (chronic obstructive pulmonary disease). A total of 10 similar consultations in the year before referral for chest X-ray]

\section{Atypical and complex presentations}

For a number of patients, atypical presentations were reported that did not lead any of the clinicians involved to initially consider lung cancer as a diagnosis. There were a variety of complex presentations and pathways, with a number of factors relating to the patient, their symptoms, and their diagnostic investigations.

'Atypical presentation. Headaches, and then leg cramps over a six month period. Then became $S O B$ and admitted as?PE ... In this case there was difficulty in establishing an early diagnoses because of the atypical presentation'.

(Case \#93: age 69, with eight consultations in the previous year)

'Difficult to follow NICE guidance on this man because he was a difficult historian (schizophrenia). Symptoms not stated'.

(Case \#77: age 44)

'Complex and atypical pathway. Difficult to determine when lung cancer symptoms started. Normal CXR (date - 11 months weeks prior to diagnosis) (GP-initiated) and again (date - 7 weeks before diagnosis) (A\&E initiated). Basal consolidation present on $C X R$ (date 2 - weeks before diagnosis) (unclear who instigated)'.

(Case \#124: age 64, presenting with a cough)

'Unusual presentation. 3 months "primary care delay" because swelling thought to be lipoma or sebaceous cyst rather than subcutaneous metastases'.

(Case \#90: age 52, presenting with

'Swelling on abdominal wall)

\section{Patients' responsibilities for delaying the diagnostic process}

GPs reported that patients themselves bore some responsibility for delays at various points in their diagnostic process. 
'Could we have diagnosed earlier? - probably not-due to patient's reluctance to present'.

(Case \#98: age 70, presenting with cough and wheezing of unstated duration)

'Probable patient delays. Failed to attend chest clinic and bronchoscopy Jan 2009 following exacerbations of COPD'.

(Case \#147: age 73, presenting with haemoptysis)

'Clinical diagnosis based upon CXR alone. Further investigations or treatment declined. Prior to diagnosis multiple rejections of admission for breathlessness, and often refused to see the doctor. Significant patient delay'.

(Case \#138: age 81, presenting with chest pain)

'The fact that patient repeatedly failed to attend follow up clinics in primary care, due to his own fears of "something being wrong" meant that the tumour progressed to the point that the patient died before treatment could be instituted'.

(Case \#49: age 67, presenting with exacerbation of COPD)

'The patient had a lifestyle which increased the likelihood of developing such pathology and a resistance to changing her behaviour and of appropriate help seeking behaviour. In the end we had to wait until she was too ill to resist intervention to access appropriate care for her problem'.

(Case \#11: age 75, presenting with gradually deteriorating health)

\section{Secondary-care responsibility for delaying the diagnostic process}

GPs reported several instances of significant diagnostic delays within secondary care.

'Delay of 31/2 months between abnormal CXR and PET scan, but which time spread to nodes and inoperable'.

(Case \#80: age 75)

'Marked secondary care delays in making the diagnosis. Indecision between locum consultants and different specialties. Radiology issues. Urgent referral not seen for a month'.

(Case \#113: age 78, presenting with shortness of breath, cough, and weight loss)
'Urgent referral was printed and faxed the same day in order to avoid delay. He was seen within 4 weeks at the secondary care but unfortunately diagnosis was missed on that occasion'.

(Case \#62: age 48, presenting with chesty cough and wheezing)

'Reviewing the records we felt that the hospital had investigated him extremely thoroughly and with no undue delay but had encountered technical difficulties'.

(Case \#63: age 69, presenting with cough and chest pain)

'Exemplary GP behaviour. Copy of CXR report sent to chest physician - exemplary. 2 months [delay] in secondary care making diagnosis because of technical difficulty with access to tumour and poor lung function'.

(Case \#137: age 76, presenting with cough with clear phlegm)

'Same day CXR request and report, and referral. USC referral then bounced by consultant who "trumped" the radiology opinion of need to refer. GP and patient only aware because GP phoned to ask what was happening. Further investigation (bloods, 2nd $C X R)$ by $G P$ and a further referral, leading to diagnosis of metastatic disease 3 months after initial referral'.

(Case \#112: age 41, presenting with cough and haemoptysis)

'Delays in reporting CXRs apparently due to lack of radiologists'.

(Case \#131: age 81, presenting with cough with haemoptysis)

\section{Quantitative analysis}

Symptoms and number of consultations before referral or admission or investigation

Of the 118 patients, $96(81.4 \%)$ presented with either respiratory symptoms or a combination of respiratory and non-respiratory symptoms and $15(12.7 \%)$ presented with solely non-respiratory symptoms. The remaining seven patients $(5.9 \%)$ were either asymptomatic or their symptoms were unclear or not stated. The non-respiratory symptoms presented are shown in Figure 3.

Primary Health Care Research \& Development 2015; 16: 436-449 
The median number of consultations before referral or admission or investigation was 1.0 , with mean of 2.04 (SD 3.38, range 0-21, IQR 0.0-3.0) (data missing on 13 patients). A total of 47 (39.8\%) patients had no consultations, 29 (24.6\%) had 1-2 consultations, $20(16.9 \%)$ had $3-5,5(4.2 \%)$ had $6-10$, and $4(3.4 \%)$ had 11 or more. Thus, exactly half of the patients who consulted before diagnosis did so three or more times.

\section{Chest $X$-rays and routes of referral and admission}

Of the 118 patients, $79(66.9 \%)$ patients had a GP-initiated chest X-ray before diagnosis and 39 $(33.1 \%)$ did not. For patients where the date of receipt of chest X-ray report was available, the median time from request to report was five days (SD 6.7), but with a range up to 24 days. A total of $23(19.5 \%)$ patients had a chest X-ray that did not initially show suspicion of lung cancer (Figure 4). The majority of patients were diagnosed after a GP-initiated referral to a chest physician; however, $10(8.5 \%)$ patients were diagnosed after referral to another specialty, and $25(21.2 \%)$ were diagnosed after a GP-initiated acute admission. Therefore, the vast majority of patients initially presented their symptoms to a GP $(106,89.8 \%)$, with 75 of

\begin{tabular}{|ll|}
\hline Back pain or slipped disc & Lump on thigh \\
Collapse with no prior symptoms & Malaise, sweats, fetor \\
Confusion, falls, bed bound & Mechanical back pain \\
Epigastric pain, dyspepsia, vomiting & Sore throat \\
Headaches [2 patients] & Right side pain \\
Hip pain & RUQ pain \\
Leg oedema & Swelling on abdominal wall \\
\hline
\end{tabular}

Figure 3 Non-respiratory presenting symptoms (15 patients) them then having a GP-initiated chest X-ray (Table 2). A minority of patients were diagnosed through other routes.

\section{Time intervals in diagnostic pathway}

Time intervals are shown in Table 3. Owing to different pathways to diagnosis and some missing dates, durations could only be calculated for less than half of the patients for four of these time periods. Only T6, T7, and T8 were calculable for three-quarters of the case reports or more. The 12 patients for whom we could not calculate a T8 (first presentation to diagnosis), all had an unclear date of first presentation or were asymptomatic. Of the 87 patients for whom time from first presentation to referral or admission was measurable, $34(39.1 \%)$ had a duration of $>31$ days.

\section{Discussion}

\section{Summary of main findings}

This paper reports a detailed analysis of the diagnostic journeys of 118 patients in Wales with lung cancer, from the perspective of GPs. Most importantly, this work clearly identifies areas that could be addressed through policy and practice initiatives in order to achieve timelier diagnoses, with potential benefits to patients. Although many diagnostic journeys occurred in a timely and appropriate manner, there was a significant proportion of patients whose diagnoses were made in a less timely fashion, sometimes with many consultations before referral for investigation or specialist opinion, or significant delays after

\footnotetext{
- Eight were reported as normal [including 1 patient who had 3 normal chest $x$-rays, the last a month before bronchoscopic diagnosis, and another had 2 normal chest $x$-rays within 2 months of diagnosis]

- Three reported no change in a pre-existing abnormality (granuloma right apex, upper lobe nodule, costophrenic angle blunting)

- Two reported other changes (Chronic Obstructive Pulmonary Disease, cardiac failure), but with no suspicion of lung cancer

- One patient had an x-ray in August 2009 that reported 'a shadow that was more conspicuous than the previous chest x-ray in November 2008', but this had not been mentioned on the November 2008 report

- Nine reported probable infection 'treat and repeat x-ray' (including four patients who had the same probable diagnosis and management recommended twice).
}

Figure 4 The 23 patients with chest X-rays that did not initially show suspicion of lung cancer 
Table 2 Diagnostic pathways

\begin{tabular}{lc}
\hline Diagnostic pathways & $n(\%)$ \\
\hline Symptoms - GP - admission & $22(18.6)$ \\
Symptoms - GP - chest X-ray - referral & $72(61.0)$ \\
Symptoms - GP - chest X-ray then radiology arranges CT and chest clinic & $2(1.7)$ \\
Symptoms - GP - chest X-ray - clinical diagnosis, no secondary-care involvement & $1(0.8)$ \\
Symptoms - GP - no chest X-ray - referral & $7(5.9)$ \\
Symptoms - GP - then self-presentation to A\&E & $2(1.7)$ \\
Via secondary-care investigation, but with some primary-care input & $5(4.2)$ \\
Via secondary-care investigation - no primary-care input & $4(3.4)$ \\
Most of diagnostic pathway made abroad (came back to UK for CT) & $1(0.8)$ \\
Opportunistic chest X-ray before treatment for rheumatoid arthritis - no primary-care involvement & $1(0.8)$ \\
Unclear & $1(0.8)$ \\
Total & $118(100.0)$ \\
\hline
\end{tabular}

Table 3 Time intervals in diagnostic pathway (days)

\begin{tabular}{lcccc}
\hline Time intervals to diagnosis & $n$ & Mean & Median & SD \\
\hline T1. Onset of symptoms to diagnosis & 48 & 204.5 & 101.5 & 264.9 \\
T2. Onset of symptoms to presentation & 51 & 107.4 & 28.0 & 196.5 \\
T3. First presentation to chest X-ray request & 72 & 19.1 & 0.0 & 33.1 \\
T4. Chest X-ray request to receipt of report & 58 & 7.1 & 5.0 & $0-730$ \\
T5. Chest X-ray report to referral & 54 & 7.8 & 1.0 & $0-152$ \\
T6. Referral to diagnosis & 92 & 37.1 & 17.5 & $0-24$ \\
T7. First presentation to referral or admission & 87 & 50.4 & 21.0 & 70.5 \\
T8. First presentation to diagnosis & 106 & 93.4 & 48.5 & $-2-79$ \\
\end{tabular}

Time intervals were calculated from the verbatim information given (Box 1 ), and in a small number of cases this resulted in paradoxically negative values; this reflects the reporting by the GPs, and the definitions of date of diagnosis used. Because they are small and infrequent, we have left these in.

referral or after chest X-ray. The reasons for this were multi-faceted and included the following: the atypical nature of some of the presenting symptoms; initial chest X-ray results being reported as normal or showing benign conditions; GPs not acting as quickly as they could to investigate or refer patients with symptoms; and a variety of other factors including patient behaviour and delays in secondary care.

The qualitative data analysis of these data showed that for many patients, the response of the GP in getting the patient into a diagnostic system was exemplary. For a smaller number, there were opportunities for the GP, in retrospect, to have investigated or referred earlier. Atypical and complex presentations have different challenges, and in some cases it remains impossible to know when the symptoms associated with ongoing lung disease become those of a lung cancer. The GPs believed that in some instances there were secondary-care issues that delayed the diagnosis. These technical diagnostic difficulties may be improved after publication of the updated NICE guidelines on the diagnosis and management of lung cancer (NICE, 2011).

\section{Strengths and limitations}

One of the main strengths of this study is that, because it replicates the methods of the NECN study, its results are directly comparable (Mitchell et al., 2009; 2013). Other strengths are the size of the dataset and the depth and quality of the data provided by participating GPs. Limitations include the fact that these data were dependent upon reporting by the GP. Therefore, there is the potential for bias in data reporting. There is also the potential for some post hoc rationalisation, after a significant diagnosis. There are several additional issues worthy of discussion. These 
include difficulty in the definition of date of diagnosis. The date of diagnosis was asked of GPs exactly as such. The data supplied could have referred to either a 'clinical' diagnosis that was based upon CT (or other imaging), a tissue diagnosis, or a full staging diagnosis. Furthermore, there may have been some difficulty in interpreting the first symptom. This is sometimes difficult to judge, from the perspective of the patient, the physician, and the researcher. The narrative nature of the data sought meant that for some patients there were missing data, especially relating to key dates in the diagnostic process. Overall, although many of our findings are similar to the English study, we regard this as a strength of our methods, and a corroboration that many of the challenges for improving lung cancer diagnosis are the same in both Wales and England, despite inherent differences in NHS structures.

\section{Discussion of the main findings within the context of the literature}

In comparison with the NECN study, the Welsh practices were very similar in terms of practice list size, rurality, and teaching and training status (Mitchell et al., 2009; 2013). Our sample of patients in the study was both similar to the NECN study and to the age at diagnosis in national data (Welsh Cancer Intelligence and Surveillance Unit, 2006). Slightly more patients in our study presented with respiratory symptoms $(81 \%)$ compared with the NECN study (74\%).

Half of the patients in the study had three or more consultations before referral or investigation. This is more than what was reported in the 2010 National Cancer Experience Survey in England. In this dataset, $33.7 \%$ of lung cancer patients had three or more consultations (Lyratzopoulos et al., 2012). Our findings highlight that lung cancer is currently difficult to diagnose, but there is the potential to act more quickly in primary care to instigate actions leading to the diagnosis.

A similar proportion of patients had a GPinitiated X-ray before diagnosis of lung cancer in this Welsh study (67\%) compared with a series reported in Devon (66\%) (Stapley et al., 2006). A total of $14 \mathrm{X}$-rays $(11.8 \%)$ of patients who were subsequently diagnosed with lung cancer were reported as normal compared with $12.8 \%$ in Devon. In our study, 12 more patients had

Primary Health Care Research \& Development 2015; 16: 436-449 abnormal but not 'suspicious of cancer' X-ray reports. From a smaller dataset, the NECN study reported that 16 out of $45(36 \%)$ chest X-rays were reported as being normal or benign. This reiterates the very important finding that a small but clinically very significant number of patients have normal X-rays before diagnosis, and that a normal $\mathrm{X}$-ray does not exclude the diagnosis.

The diagnostic pathways reported here are similar to those reported by Barrett and Hamilton (2008) in their cohort from Devon. They reported the proportion of diagnoses made after outpatient referral as $61 \%$, emergency referral as $23 \%$, and asymptomatic as $11 \%$. Our data reported $61,18.6$, and $9.3 \%$. Our findings reiterate the fact that GPs have the potential to improve the timeliness of their referrals for most patients who are diagnosed with lung cancer.

Our overall time from symptom onset to diagnosis was a median of 101.5 days; this compares with 108 days in the Denmark study (Hansen et al., 2011). The NECN study reported time from relevant symptom presentation to referral or acute admission as a mean of 59 days (Welsh data mean of 50 days) and a median of 21 days (Welsh median 21 days), with $(59 \%) 31$ days or less (Welsh data $61 \%)$. The figure from a Scottish study was a median of 11 days (Baughan et al., 2009). The findings reported from secondary analysis of data from the national survey of cancer patients (Allgar and Neal, 2005) are also similar to the data presented here - a median time from referral to diagnosis of 21 days (Welsh data 16 days). In comparison with the Scottish data (based on 981 patients, exact method of asking the questions not stated), the time from first symptom to presentation was longer in Wales (median 28 days) compared with Scotland (median 9.5 days), which suggests that there is more research required into patient awareness and presentation behaviours. Our qualitative findings of a small number of significant delays in secondary care are corroborated by data from Denmark, demonstrating a median of 27 days from being seen in secondary care to diagnosis (Hansen et al., 2011). The findings highlight the long time intervals in diagnosis and the potential to reduce these.

A small qualitative study from New Zealand has recently reported that reasons for diagnostic delay in lung cancer are complex and multi-factorial (Walton et al., 2013). Mitchell et al. (2013) report complexity in a number of cases, often related to 
co-morbidity or to symptoms suggestive of another malignancy. Our findings add to this by demonstrating the range of difficulty and complexity, and from the perspective of the GP.

The significant event process meant that participating GPs learned much about improving their lung cancer diagnoses, as well as about the diagnostic process and communication around this. The process led to significant changes in the diagnostic process, and it was felt to be a very valuable tool, with many additional benefits (Neal et al., 2010).

\section{Implications for policy, practice, and research}

As stated above, the most important implication of this work is that it clearly identifies areas that could be addressed through policy and practice initiatives to achieve timelier diagnoses with potential benefits to patients. The implications from our findings for GPs are that, although they should continue to comply with guidelines, they should be vigilant when faced with atypical symptoms and consider lung cancer as a potential diagnosis. They should also be vigilant with patients with ongoing symptoms and a normal chest X-ray. There are also implications for secondary care to ensure that processes are in place to streamline diagnostic procedures. Finally, we believe that there is a need for more research to develop and evaluate interventions to increase patient awareness of symptoms and to hasten the diagnostic process for patients with suspected lung cancer.

\section{Acknowledgements}

The project was funded by the Welsh Government through their funding of the Cancer National Specialist Advisory Group, Public Health Wales (formerly Cancer Services Co-ordinating Group) as part of the work of the all Wales Lung Cancer Advisory Group. The work involved a partnership between the CSCG, the School of Postgraduate Medical and Dental Education (Deanery), Cardiff University, and the North Wales Centre for Primary-Care Research. RDN receives funding from Public Health Wales and Betsi Cadwaladr University Health Board. The authors thank the general practitioners who participated in this study. In particular the authors acknowledge the support from the regional cancer network directors, Damian Heron (North Wales), Glynis Tranter (South West Wales), Hywel Morgan (South East Wales), and the GP leads namely Dr Rhys Davies (North Wales), Dr Bridget Gwynne and Ms Janet John (South West Wales), and Drs Liam Taylor and Mark Smithies (South East Wales). The authors also thank Professor Greg Rubin, Durham University, for advice on planning for this project.

\section{Conflicts of Interest}

None.

\section{Ethical Standards}

In England, the National Research Ethics Service advised that SEA for cancer is considered a service audit, providing secondary analysis for GPs and does not require ethical review.

\section{References}

Allgar, V.L. and Neal, R.D. 2005: Delays in the diagnosis of six cancers: analysis of data from the national survey of NHS patients: cancer. Br J Cancer 92, 1959-70.

Barrett, J. and Hamilton, W. 2008: Pathways to the diagnosis of lung cancer in the UK: a cohort study. BMC Fam Pract 9, 31.

Baughan, P., O'Neill, B. and Fletcher, E. 2009: Auditing the diagnosis of cancer in primary care: the experience in Scotland. Br J Cancer 101, S87-91.

Corner, J., Hopkinson, J., Fitzsimmons, D., Barclay, S. and Muers, M. 2005: Is late diagnosis of lung cancer inevitable? Interview study of patients' recollections of symptoms before diagnosis. Thorax 60, 314-19.

Elliss-Brookes, L., McPhail, S., Ives, A., Greenslade, M., Shelton, J., Hiom, S. and Richards, M. 2012: Routes to diagnosis for cancer - determining the patient journey using multiple routine data sets. Br J Cancer 107, 1220-26.

Gale, N.K., Heath, G., Cameron, E., Rashid, S. and Redwood, S. 2013: Using the framework method for the anlaysis of qualitative data in multi-disciplinary research. BMC Med Res Methodol 13, 117.

Hamilton, W.T. 2009: The CAPER studies: five case control studies aimed at identifying and quantifying the risk of cancer in symptomatic primary care patients. $\mathrm{Br} J$ Cancer 101, S80-86.

Hansen, R.P., Vedsted, P., Sokolowski, I., Søndergaard, J. and Olesen, F. 2011: Time intervals from first symptom to treatment of cancer: a cohort study of 2,212 newly diagnosed cancer patients. BMC Health Serv Res 11, 284.

Keeble, S., Abel, G.A., Saunders, C.L., McPhail, S., Walter, F.M., Neal, R.D., Rubin, G.P. and Lyratzopoulos, G. 2014: 
Variation in promptness of presentation among 10,297 patients subsequently diagnosed with one of 18 cancers: evidence from a national audit of cancer diagnosis in primary care. Int $J$ Cancer. Advance Online Publication. $1 ; 135(5), 1220-28$.

Lyratzopoulos, G., Abel, G.A., McPhail, S., Neal, R.D. and Rubin, G.P. 2013: Measures of promptness of cancer diagnosis in primary care: secondary analysis of national audit data on patients with 18 common and rarer cancers. Br J Cancer 108, 686-90.

Lyratzopoulos, G., Neal, R.D., Barbiere, J.M., Rubin, G. and Abel, G. 2012: Variation in the number of general practitioner consultations before hospital referral for cancer: findings from a national patient experience survey. Lancet Oncol 13, 353-65.

Mitchell, E., Macleod, U. and Rubin, G. 2009: Cancer in primary care. An analysis of significant event audits (SEA) for diagnosis of lung cancer and cancers in teenagers and young adults 2008-9. Glasgow and Durham: Universities of Dundee.

Mitchell, E., Rubin, G. and Macleod, U. 2013: Understanding diagnosis of lung cancer in primary care: qualitative synthesis of significant event audit reports. $\mathrm{Br}$ J Gen Pract $63,37-46$.

National Institute for Health and Clinical Excellence. 2005: Referral guidelines for suspected cancer. London: NICE.

National Institute for Health and Clinical Excellence. 2011: The diagnosis and treatment of lung cancer (update of NICE clinical guideline 24). London: NICE.

National Patient Safety Agency. 2006: Seven steps to patient safety for primary care. London: NPSA. Retrieved 1 June 2013 from http://www.npsa.nhs.uk

Neal, R.D., Din, N.U., Hamilton, W., Ukoumunne, O., Carter, B.R., Stapley, S. and Rubin, G.P. 2013: Comparison of cancer diagnostic intervals before and after implementation of NICE guidelines: analysis of data from the UK general practice research database. Br J Cancer 110, 584-92.
Neal, R.D. and Muers, M. 2009: Symptoms, assessment and guidelines for referral. In Hunt, I. and Treasure, T., editors, ABC of lung cancer. London: Blackwell, 1-5.

Neal, R.D., Robbé, I.J., Lewis, M., Williamson, I. and Hanson, J. 2010: Significant events audit of lung cancer in primary care in Wales. Cancer Services Co-ordinating Group, Cardiff 1, 21-23.

Neal, R.D., Rogers, T. and Hamilton, W. 2014: Easily missed? Lung cancer. BMJ 349, g6560.

NHS Information Centre. 2011: Retrieved 1 June 2013 from http://www.ic.nhs.uk/services/national-clinical-audit-supportprogramme-ncasp/cancer/lung

O'Dowd, E.L., McKeever, T.M., Baldwin, D.R., Anwar, S., Powell, H.A., Gibson, J.E., Iyen-Omofoman, B. and Hubbard, R.B. 2014: What characteristics of primary care and patients are associated with early death in patients with lung cancer in the UK? Thorax, first published online 20 October 2014, doi 10.11136/thoraxjnl-2014-205692.

Office for National Statistics. 2010: Cancer and mortality in the United Kingdom 2005-2007. Retrieved 1 June 2013 from http://www.statistics.gov.uk/pdfdir/canuk0810.pdf

Stapley, S., Sharp, D. and Hamilton, W. 2006: Negative chest $\mathrm{X}$-rays in primary care patients with lung cancer. Br J Gen Pract 56, 570-73.

Walton, L., McNeill, R., Stevens, W., Murray, M., Lewis, C., Aitken, D. and Garrett, J. 2013: Patient perceptions of barriers to the early diagnosis of lung cancer and advice for health service improvement. Fam Pract 30, 436-44.

Weller, D., Vedsted, P., Rubin, G., Walter, F., Emery, J., Scott, S., Campbell, C., Andersen, R.S., Hamilton, W., Olesen, F., Rose, P., Nafees, S., van Rijswijk, E., Muth, C., Beyer, M. and Neal, R.D. 2012: The aarhus statement: improving design and reporting of studies on early cancer diagnosis. Br J Cancer 106, 1262-67.

Welsh Cancer Intelligence and Surveillance Unit. 2006: Trachea, bronchus and lung cancer in Wales. Cardiff: Welsh Cancer Intelligence and Surveillance Unit. 


\section{Appendix A - data collection template}

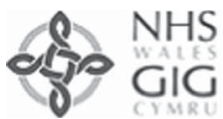

Cancer Significant Event Audit (2010) REPORT TEMPLATE



\begin{tabular}{|l|l|}
\hline Diagnosis & \\
\hline Date of diagnosis & \\
\hline Age of patient at diagnosis & \\
\hline $\begin{array}{l}\text { Is the patient currently alive? } \\
\text { (if not alive, please give date of death) }\end{array}$ & \\
\hline Date of SEA meeting & \\
\hline
\end{tabular}

\footnotetext{
1. What happened?

Describe the process to diagnosis for the patient, including dates of consultations, referral and diagnosis. Consider, for instance, the key consultation at which diagnosis was made, consultations for this patient in the practice in the year prior to diagnosis and the referral process. How often had the patient been seen? Had he/she been seen by the out of hours service, at A\&E or in secondary care clinics? Was there any delay on the part of the patient in presenting with their symptoms?
} 


\section{Richard D. Neal et al.}

\section{Why did it happen?}

Reflect on the process of diagnosis. Was this as good as it could have been? If so, what were the factors that contributed to speedy and /or appropriate diagnosis in primary care? If there was some delay in diagnosis, what were the underlying factors that contributed to this? Were reasons for any delay acceptable or appropriate?

\section{What has been learned?}

Describe the discussion at the team meeting. Demonstrate that reflection and learning have taken place on an individual or team basis and that relevant team members have been involved in considering the process of diagnosis. Consider, for instance: a lack of education or training; the need to follow systems of procedures; the importance of team working or effective communication. Consider the role of the NICE Referral guidelines for suspected cancer and their usefulness to primary care teams.

Primary Health Care Research \& Development 2015; 16: 436-449 


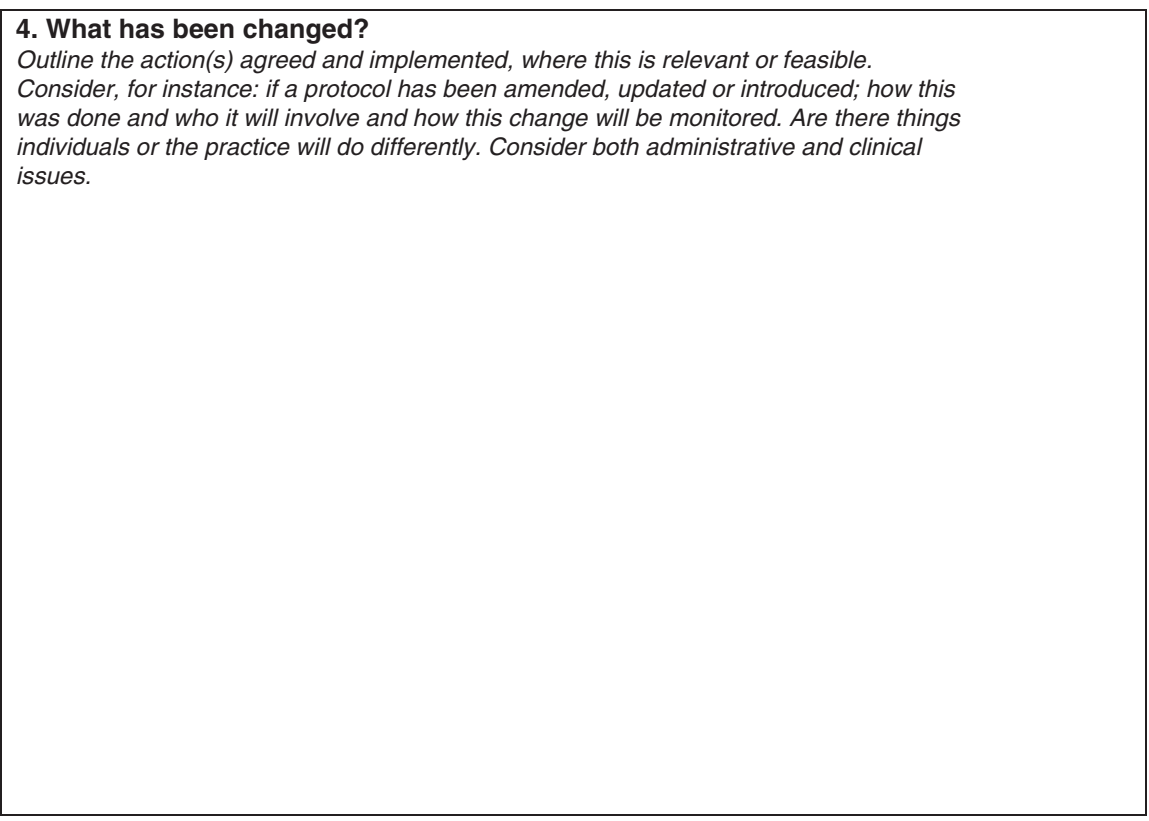

What was effective about this SEA?

Some information about your practice

How many patients do you have?

Which of the following best describes your practice?

Is your practice a training practice?

Do you teach medical students?



Yes

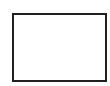

25005000

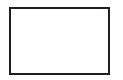

$>5000$

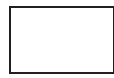

Semirural

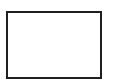

Rural

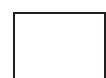

No

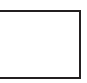

No

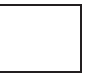

